\title{
Software Architectures for Smart Applications in the Management of Chronic Diseases: A Study of Reversibility of Diabetes 2
}

\author{
Mehdi Tarabi \\ Department of Science and Industry Systems \\ University of South East Norway \\ $\underline{\text { Mehdi.tarabi@student.hbv.no }}$
}

\begin{abstract}
The paper proposes a software architecture for applications which use the reasoning upon SWRL enabled OWL ontologies and SQL like retrievals as core computational models. The application assists patients affected by diabetes 2 , to personalize the reversibility of the condition through the diet and life style changes. The novelty is in (a) the deployment of SWRL enabled OWL ontologies in the management of data related to the personalisation of reversibility of diabetes 2 and in (b) the proposed software architecture, which contains and manipulates the SWRL enabled OWL ontology and SQL databases at the same time and transparently. The application, which has been implemented within the Java environment and NetBeans, is reusable in any other problem domain when the personalization of healthcare delivery is required. The proposed architecture also generates applications within Android environments without changing its style and the computational models.
\end{abstract}

\section{Introduction}

Personalized $(1,2)$ and precision $(3,4)$ medicine are key terms which dominate our delivery of healthcare. The paradigm shift from personalized to precision medicine has already taken its momentum $(5,6)$ and, it is impossible to create new software solutions which support modern healthcare delivery without looking at the power of personalization and precision $(7,8,9)$. We also talk about new paradigms of participatory, personalized and preventive medicine, which will not only affect the practice of delivering individual healthcare $(10,11)$, but it could also secure affordable and efficient healthcare across the globe $(12,13)$. In this research we looked at the problem of addressing the management of chronic diseases, by developing software applications, which can personalize the support patients may need when living with chronic conditions $(14,15,16)$. We are particularly interested in the management of diabetes, due to many initiatives of governments, which try to

\author{
Radmila Juric \\ Department of Science and Industry Systems \\ University of South East Norway \\ rju@usn.no
}

address its step rise across the world. In the last 5 years we witnessed a proliferation of information promoting the reversibility of diabetes 2 in particular $(17,18,19)$. There are many books on the Amazon and blogs and information from diabetes societies on the Internet, which inform people about the reversibility of diabetes 2 . Almost all of them focus on the power of diet and life style in order to address the problem $(20,21,22)$.

Therefore, we have been motivated to propose a software solution for personalized management of this condition and assist patients to make decisions on personalized changes in their life style and diet. We wanted to develop a lightweight software application, which would be deployable on smart devices, but would give an instant, i.e. an ad-hoc advice to a particular patient on how to personalize the reversibility of diabetes 2 . This can happen when either the patient is motivated to take an action or when his/her clinical data "signals" that the rising level of glucose in blood needs urgent attention. It is obvious that such software applications could not be developed using traditional methods of storing all relevant information on reversibility of diabetes 2 in big repositories and knowledge bases and retrieving data from them. Personalization of information delivered to such patients must conform to two important requirements:

A) Advice on diet and life style changes must be articulated in real time, when either the patient current clinical data (available on an ad-hoc basis) or patient requests trigger it;

B) We should allow constant changes to be inferred within our software solution, which would address either patient current clinical status or advances in research on reversibility of diabetes 2. They should be available at any time without changing the design of the software solution.

It is quite difficult to create such software applications. Apart from the traditional processing with well-known structured SQL data repositories we will have to perform reasoning in order to address A) 
and B). The decision to stay outside artificial intelligence algorithms for performing reasoning, was based on our previous experiences of running SWRL rules for performing reasoning as a software engineering solution $(23,24,25,26,27)$. Therefore the deployment of Semantic Web Technologies (SWT) (28) and their languages: OWL (29) and SWRL (30) has become our obvious choice. However, before we assume that the software solution, which satisfies A) and B) could be deployed with various technologies, we had to create a software architectural model, which would guarantee the deployment and reusability of our proposal across platforms and technologies. We also wanted to see if our solution would work for personalizing the management of other chronic diseases in real time.

The paper is organized as follows.

In section 2 we list related work which might be applicable to this research and comment on our contribution towards it. In Section 3 we perform UML modelling of a generic software architecture which should generate our software solution. In Section 4 we define the computational model which is in the heart of the proposed architecture: it delivers the functionality of the software application, but uses two different computing paradigms. The traditional SQL database retrievals and updates upon the patent's demographic and clinical data is similar to software applications found in any patient record systems. However, a completely different computational model, which needed more explanations, is given in section 5. It focuses on the creation of SWRL enabled OWL ontologies and define OWL concepts (classes, individuals and constraints) and reasoning rules which perform the computation. In section 6 we show user interfaces and results of running the application with both computational models. In section 7 we debate the value of the architectural proposal, the efficiency and reusability of the application generated from it, and point towards future work which could secure the commercialization of such solutions in personalized healthcare in general.

\section{Related Work}

Due to restrictions of space, we choose to comment on two types of peer-reviewed papers, which either use SWT and ontologies in healthcare or apply them in the management of reversibility of diabetes 2. Papers $(31,32,33,34,35)$ are examples of using OWL ontologies and SWT in healthcare. They range from expert systems for diagnosing diabetes and supervision of patients with acute cardiac disorders to solutions for managing clinical practice guidelines and managing biomedical data interlinked with complex semantic relationships. However, all these examples show the use of SWT through the creation of formal ontologies. We would like to draw reader's attention that our solutions will not use SWT and OWL ontologies in the same manner, because formal ontologies would not be able to address A) and B) from Introduction $(26,27)$. We need SWRL enabled OWL ontologies to help us in computing an instant answer to patients regarding the personalized reversibility of diabetes 2 . This means that we will not be able to cumulate excessive knowledge in our OWL ontologies because it will have impact on the personalization. Patient circumstances can constantly change, even throughout the day, and therefore we need to manipulate the semantics of the moment in which an ad-hoc answer is expected from the application.

There are a few publications, which approach our ideas of using the SWT in the management of diabetes 2. The authors of (36) show OWL representation for the knowledge modelling and creating answers to patient's questions regarding their diabetes treatment planning within homes. They use formal and domain ontologies and prepare treatment plans through various stages of data processing. It is difficult to see the exact level of personalization they could achieve in their application. In (37) we have a solution which creates individual (personalized) ontologies for a patient and then match them with existing knowledge stored in formal ontologies, in order to advice patients on their diet and exercise management. These applications, however successful they may seem, are heavy-weight and require significant computational management which is not efficient on smart devices. Finally the authors of (38) propose a good example of balancing all relevant knowledge on diabetes 2 in ontologies, and resolving the issue of managing the semantics of advices available in them. None of these three solutions merge the traditional processing of clinical data stored in SQL databases with reasoning through SWRL enabled OWL ontologies in order to personalise the reversibility of diabetes 2 . Finally the authors of (39) give an outline of mobile applications available on the market which address diabetic patient life style management, but none of them deal with the personalized aspect of the management.

\section{UML Modelling of the Proposal}

UML modelling practices require depicting the functionality of the application we wish to develop by creating use cases, discovering main modelling abstractions from them in sequence diagrams, and conceptualizing objects from sequence diagrams into classes and software components, which comprise an 
architectural model of the application. Figure 1 shows three major functionalities of the application: the user is able to retrieve patient demographic and clinical data (RPD), update patient glucose level (UGL) whenever needed, and request the creation of a particular meal (CM), based on the current glucose level readings.

The modelling of the Application would require developing a separate sequence diagram for each use case from Figure 1.

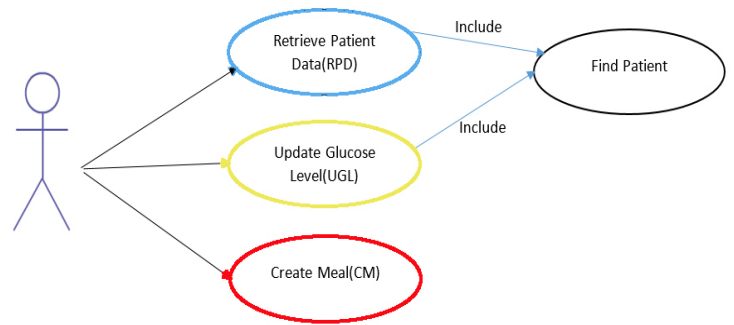

Figure 1 Use case Diagram for the Application

Figure 2 illustrates one sequence diagram in which the main abstraction, are derived from the CM use case. The objects revealed in Figure 2 are selfexplanatory: CM.UI is a user interface object which allows the user to request the creation of a "meal", CM.SQL object will contain an SQL statement which will retrieve a current glucose level reading from the PATIENT object (i.e. records in a SQL like database which stores patient demographic and clinical data). CM.SWRL object contains a SWRL rule, which would take data available within the OWL ontology: current glucose readings (OWL-Patient_glucose) and food available (OWL-Food) and select from OWLFood only individuals which can constitute a "meal" (OWL-Good food).

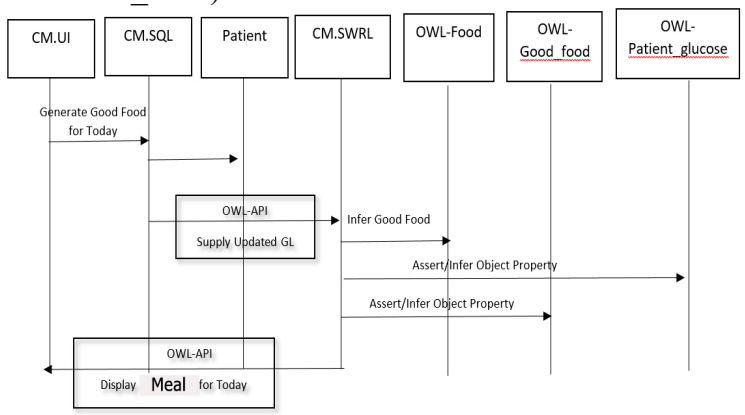

Figure 2: A Sequence Diagram from CM Use Case

It is important to note that the use case models generate a set of objects, conceptualized into UML classes/ software components, in order to create a software architectural model. Figure 1 defines the functionality expected to appear in the software architecture, but sequence diagrams bring software components which will make up the architecture.

\subsection{Proposing Software Architecture}

Figure 3 is the Software Architecture, derived from all use cases and sequence diagrams, which underpins the exact application architecture and illustrates the way functionalities from Figure 1 will have to be implemented. It also shows which type of data is processed in the Application: structured and SQL like clinical and patient demographic data and individuals of OWL ontologies (OWL-Patient glucose, OWL-Food and OWL-Good_food) in order to secure the reasoning with SWRL and create a "meal".

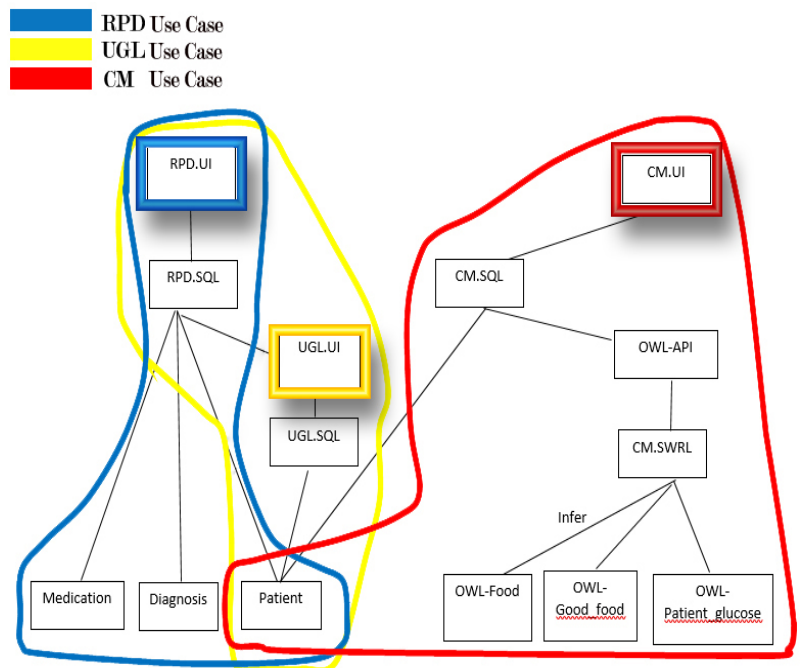

Figure 3: Software Application Architecture

Figure 3 highlights two aspects of the architecture:

a) Data processing using SQL is separated from the processing with SWRL (reasoning) and therefore the deployment of the components from Figure 3 would need technological support in order to move across different types of computations.

b) The component which contains data relevant to PATIENT is reused. Some part of PATIENT data is essential in SQL like processing (e.g. updating glucose level) and some parts (e.g. current glucose level) are needed for reasoning with SWRL and creating a "meal".

Software Architecture from Figure 3 can only be deployed if we attach to each of its software components technology specific requirements for the implementation. Figure 4 illustrates exactly what is needed in order to deploy the solution from Figure 3:

(i) The main functionality has been deployed through an IDE, such as NetBenas, which utilizes 
JSP and servlet technologies to manipulate data stored in SQL server and consequently manage the patient's demographic and clinical data.

(ii) The window towards the reasoning part of the Application is defined as OWL-API, which enables copying of the result of the retrieval of current glucose level from the PATEINT data (CM.SQL servlet) to become an individual of the OWL-Patient_glucose class and prepare OWL ontology for the reasoning with SWRL (CM.SWRL) in order to create a "meal".

(iii) The results of reasoning (the individuals of OWL-Good food class) could be displayed within the CM.UI interfaces using the same OWL-API.

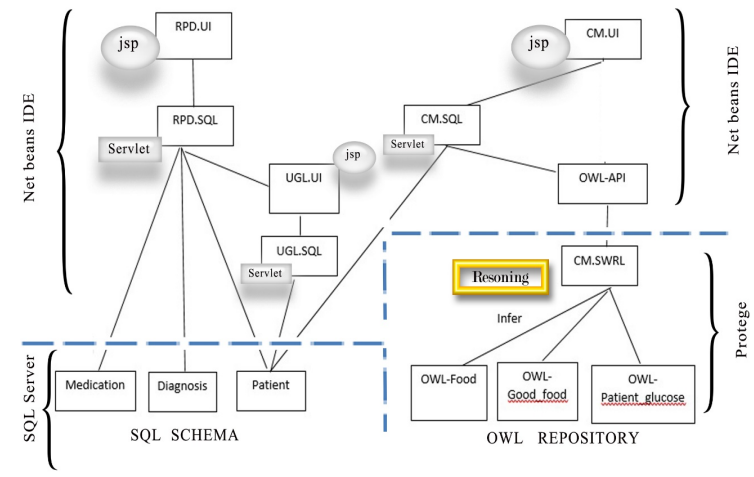

Figure 4. Technology Specific Software Architecture

The illustration of the architecture from Figure 4 is in Figure 5. We could see that the personalization of creating a "meal" may happen at any moment: The application does not create a uniform "meal" for various glucose levels: the creation of a "meal" is triggered by a particular glucose level reading, which is retrieved form the patients' clinical data and updated whenever a patent wishes to do so. Triggering the creation of a "meal" could be automatic, by the application (whenever glucose level readings change), or by the patient who may require a "meal" after checking his glucose level reading. It is important to note that the issue of privacy of patient records could be address with known role based accessed control, which has not been shown here. However, the box on the bottom left Figure 5 shows exactly which data form patient records can be retrieved in order to request a "meal" though the reasoning.

\section{Computational Model for Application}

The computational model, defined in Figures 3 and 4 consists of two parts. The first part uses SQL like statements and data stored in SQL schema within a server and therefore it is similar to most of the existing computations, which manage patient records.
The second part uses SWRL enabled OWL ontologies for the purpose of reasoning.

The similarity between our computational SQL model and traditional software applications, which manage patient records, is in

- the way the data is stored: in structured repositories, associated with databases and

- the existence of SQL like processing of the structured data, often connected with inserts, updates and retrieval.

The emphasis is on structured data definition and their SQL manipulation. These computations will be able to manipulate any demographic and clinical data from patient records and prepare it reasoning.

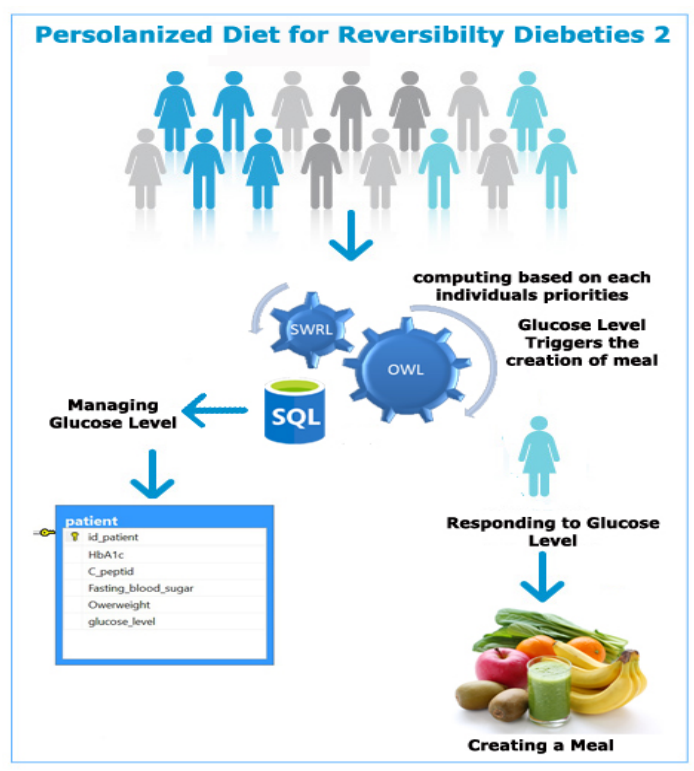

Figure 5: Illustration of the Architecture

It is important to note that the SQL schema within the SQL server in Figure 4 is rather independent from the rest of the application, which means that we can, within the same architectural model, use various and existing solutions for manipulating patient records available in real life. The SQL schema illustrated in Figures 3 and 4 has been simplified for the purpose of securing essential clinical data for reasoning, but a full scale commercial database schema of patient records will work here equally well.

\section{Computational Model for Reasoning}

The computational model based on reasoning with SWRL requires a completely different formatting of data and its processing. The data is stored in OWL ontologies which require a model of its basic classes and their individuals. It also needs definitions of constraints imposed on these classes and individuals 
which strengthen the semantics they stored in these ontologies.

Therefore in the next few subsections we describe the OWL model, define constraint in the form of object properties and specify the SWRL rule which would perform reasoning and therefore provide the results of this type of computations. Consequently we name such ontologies SWRL enabled OWL ontologies.

It is important to note that the definition of the reasoning, as suggested in Figure 4 happens in the ontological editing tool Protégé and thus the content of this subsection is Protégé and OWL specific.

\subsection{The OWL Model}

Figure 5 shows basic classes of the OWL model. Their presence is indicated in lower right part of the architecture from Figures 4 and 5, but a full scale OWL classes from Figure 6 reflect the semantics needed to be modelled. We need to know the roles of the classes, their individuals and constraints within the SWT paradigm in order to secure reasoning.

Classes in Figure 6 show that individuals of Food and Glusoce_Level classes should be important when reasoning in order to answer, "which meal should be prepared". The same applies to individuals of Physical Activities and Glusoce Level: they should be related in order to create an Exercise (program), which would be a response to the latest (or current) glucose level readings.

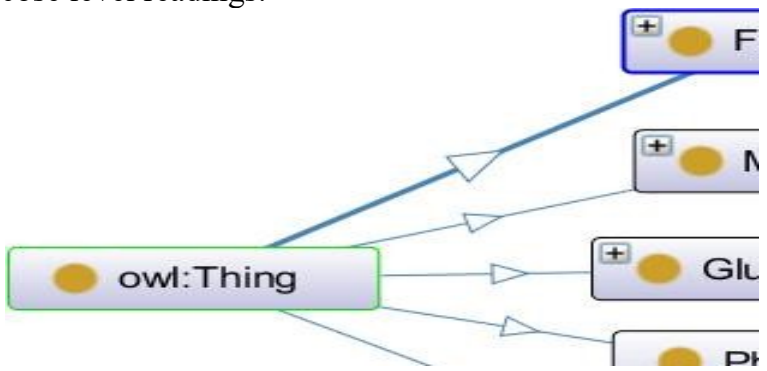

Figure 6: Classes of the OWL model

It is obvious that our reasoning will filter individuals from Food and Physical Activity classes and infer them into Meal and Exercise classes. However, the inference is strictly dependent on both: OWL constraints and the definition of the reasoning process. They should guarantee a universal SWRL rule, possibly without literal values, which would run unchanged regardless the changes of the value of individuals and asserted or inferred constraints.

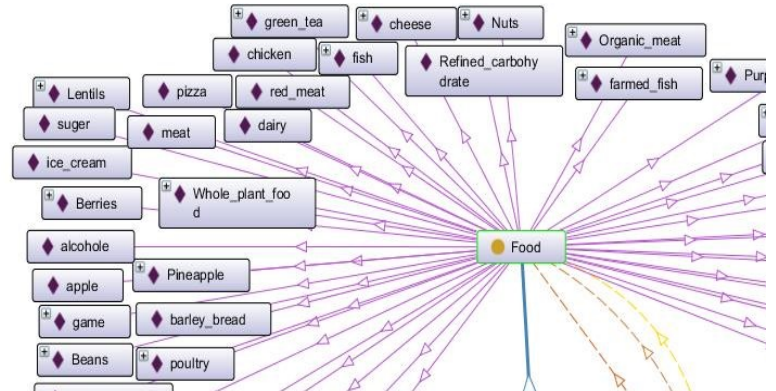

Figure 7: Individuals of Food class

For testing purposes we asserted various individuals in the Food class. They are shown in Figure 7 . They have covered a variety of food and ingredients which could be used when creating a meal. This is the only class in the OWL model which can be heavily populated with individuals.

\subsection{OWL Constraints}

Table 1 shows a selection of object properties imposed on individuals of the OWL model. The left most column in the table represents a domain and right most is a range class for each object property. We show only two object properties, which define "which food should be suitable for which glucose level reading".

Table 1: An illustration of Constraints in the OWL Model

\begin{tabular}{|c|c|c|}
\hline $\begin{array}{l}\begin{array}{l}\text { Glucose_Level } \\
\text { (domain) }\end{array} \\
\end{array}$ & Object Property & $\begin{array}{l}\begin{array}{l}\text { Food } \\
\text { (range) }\end{array} \\
\end{array}$ \\
\hline \multirow[t]{8}{*}{ gl1 } & is_good_for_GL1 & Poultry \\
\hline & & $\begin{array}{l}\text { Whole plant } \\
\text { food }\end{array}$ \\
\hline & & $\begin{array}{l}\text { Beverage } \mathrm{W} / \mathrm{O} \\
\text { sugar }\end{array}$ \\
\hline & & Pineapple \\
\hline & & Farmed fish \\
\hline & & Fish \\
\hline & & Organic meat \\
\hline & & Game \\
\hline \multirow[t]{10}{*}{$\mathrm{gl3}$} & is_good_for_GL3 & Cheese \\
\hline & & Organic meat \\
\hline & & Purple potatoes \\
\hline & & Chocolate \\
\hline & & Berries \\
\hline & & $\begin{array}{l}\text { Broccoli } \\
\text { sprouts }\end{array}$ \\
\hline & & Beans \\
\hline & & Green tea \\
\hline & & Fish \\
\hline & & Lentils \\
\hline
\end{tabular}

Therefore for GL1, which is an individual of Glucose_Level class there is a relationships with 
various individuals of Food class. The extraction of data, asserted within the OWL ontology and placed in Table 1 is for illustration only. A full scale of object properties have to be defined within the OWL model for all individuals, which "relate" through that property. Finally, the decision on suitability of any individuals of Food class to participate within any object property does not have to be asserted: we can infer automatically through SWRL reasoning any

Food(?a)^is_good_for_GL3(gl3,?a)->Meal(?a)

relation, which is defined in Table 1 . We should also draw the reader's attention that individuals of Glucose level class are enumerated and they represent a RANGE of values which may appear as patient's current glucose level readings. This means that various readings, which belong to the same range should be related to the same recommended food in order to create a "meal".

\subsection{The Reasoning Process}

The reasoning process performed with SWRL depends on the way OWL model and its constraints have been defined. In this particular case we perform filtering of Food individuals according to the potential inference of object properties. Therefore Figure 8 is a simple illustration of such a process. After running a SWRL Rule(s) upon individuals of Food and Glucose_level classes, using previously defined object properties between these two classes, some individuals of class Food will be copied into the Meal class. This inference will secure that the software application gets the individuals of the Meal class and creates a meal for a particular glucose level reading which was passed to the OWL ontology from the patient clinical data stored within an SQL server. In Fig. 8 only classes involved in the reasoning are shown: all other OWL concepts have been ignored.

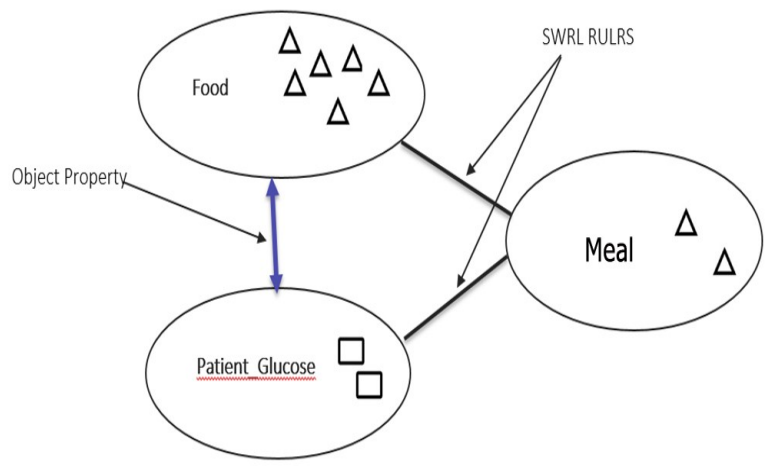

Figure 8: The Reasoning Process

\subsection{The SWRL rule}

Figure 9 shows the SWRL rule defined according to the reasoning process rorm Figure 8 . The rule has a very important characteristic, which makes it quite powerful. It is generic: the code remains unchanged even if the content of individuals of OWL classes and their object properties change. Thus the dynamic inference of object properties will not affect the format of the rule. The rule reasons upon the OWL concepts and concludes exactly "which meal to prepare for glucose level reading in range GL3."

Figure 9. SWRL rule for creating a meal

\section{Running the Application}

In this section we show user interfaces defined in the IDEs environment of the architecture in Figure 4 and screenshots of the Protégé editing tool where the reasoning has been performed. Therefore Figure 10 is a uniform interface which collates RPD.UI, UGL.UI and CM.UI from Figure 4.

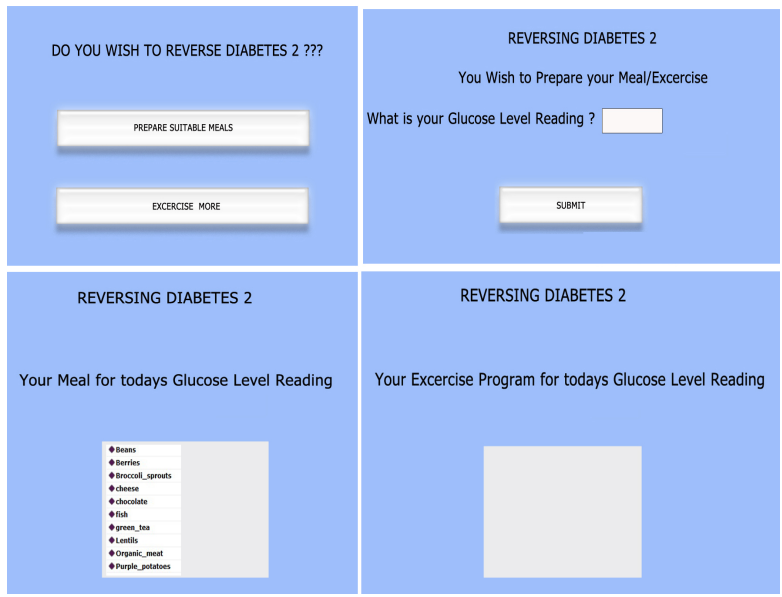

Figure 10 Interfaces for the Application

In the left top corner we allow users to choose how they wish to address the current glucose readings: either through preparing a "meal" or including an exercise program. In this paper, we illustrate computational model, which deals with the preparation of a "meal", but a full-scale application which includes a regime of exercises will have the same computational model. The only difference will be the inference of correct object properties and the assertion of individuals in Physical Activity class. The right top corner in Figure 10 allows updating of Glucose level reading within the SQL database. The bottom parts of the figure show the result of reasoning after running the SWRL rule from Fig. 9. 
Most of the computations in this application are performed within the Protégé environment, but the result of the reasoning is transferred to the application's interface using OWL-API. Figures 11 and 12 show two screenshots from the Protégé.

Figure 11 shows the execution of SWRL rule copied in Figure 9 and Figure 12 shows a list of individuals from class MEAL which were inferred after the SWRL rule was run. These individuals are also visible in the left lower part of the UI form Figure 10.

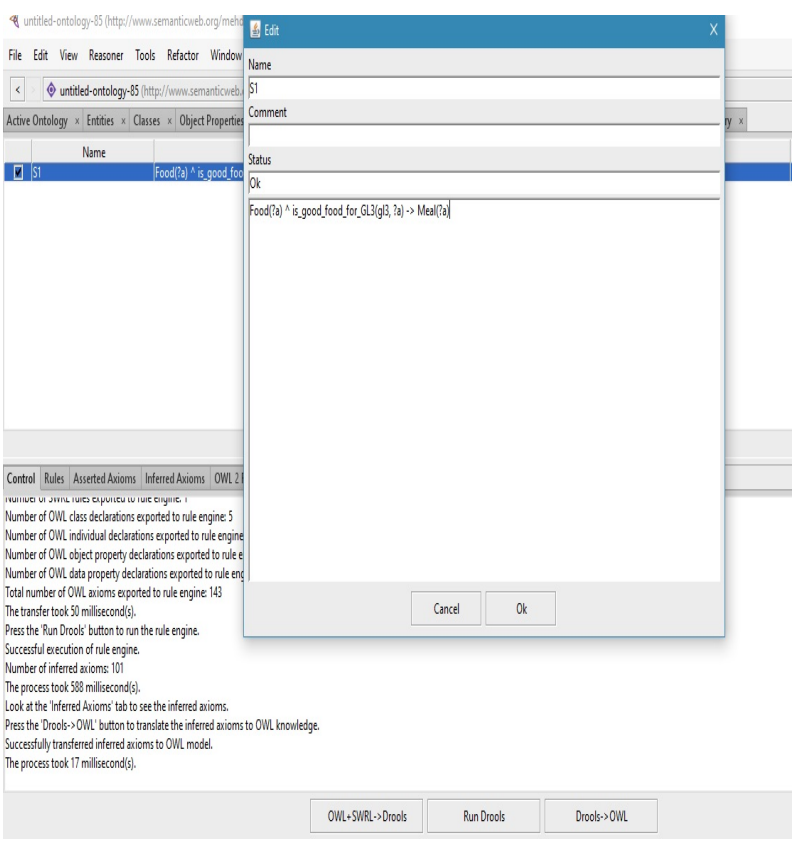

Figure 11: The SWRL Rule in Protégé

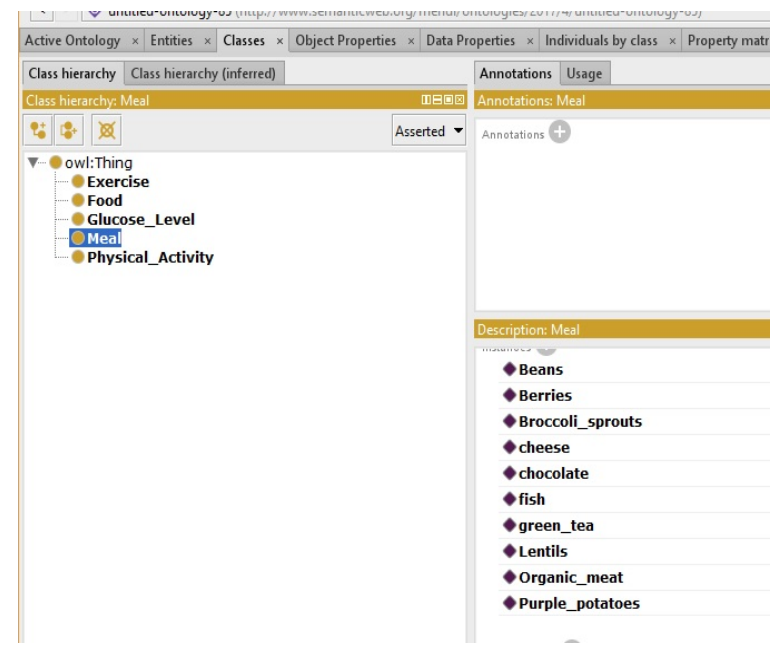

Figure 12 Individuals of the Meal Class

\section{Conclusions}

\subsection{Debating the Architectural Proposal}

One of the most important goals of this research was to create a generic and reusable software architectural style (40), which can be reused in cases where SQL like data processing has to be joint with reasoning upon OWL ontologies. The goal has been achieved for two reasons:

(a) the architecture in Figure 4 separates these two types of computations, making them independent, but at the same time joins their result through plug inns (OWL-API), interfaces (Figure 10) and sharing of data (PATIENT data from SQL schema). This means that the flexibility of the architecture is built-in. We can change the processing of patient records and use the same reasoning with the OWL model, as long as access to clinical data and patient records are secured. We can leave the management of patient records intact and update OWL concepts to accommodate new results of research in reversibility of diabetes: the architectural model will remain the same.

(b) the architecture follows the Model-ViewController pattern, which was underpinned by component based Java technologies, noted in Figure 4. It also resembles a software architecture, which proved to be successful and efficient for the implementations in Android environments (41).

Therefore, "jumping" from strictly structured data processing in SQL to reasoning with description logic and SWT and back, does not represent a barrier for software applications if its software architectural components are layered and connected as in Figures 3 and 4. NetBenas, an Integrated Development Environment (IDE) secures adherence to the MVC pattern and the Android studio offers the same option. Therefore converting the application developed here into a smart phone App should not be a problem, as long as the same software architecture is being used. Finally, the architecture has been derived from the traditional UML modelling practices, following agile development principles which also secures its longevity through the layering and grouping of software components as in Figure 4. If for any reason technology specific components in that figure will change, the architecture from Figure 3 should remain the same: numerous plug-ins, which exist in today's heterogeneous software developments, are very often feasible solutions when moving from one technology to another.

\subsection{Evaluation of the Application}

Apart from making sure that the application addresses the reversibility of diabetes 2 , and follows 
the architecture from Figure 4, we also wanted to develop a software solution which

a) works for some other chronic diseases where changes in lifestyle of patients are advisable and

b) helps the affected population to be guided individually with personalized and an ad-hoc decision making.

This means that we needed a flexible, lean, and easy to implement software solution, which will assist an individual at any time to manage the reversibility of diabetes 2 and still provide the possibility of using clinical data as much as required. Our SWRL enabled OWL ontologies secured exactly this outcome. We do not build big, formal ontologies here: we use OWL and SWRL for instant decision making, which can be executed as many times as needed, i.e. whenever SWRL is triggered by the changes of the glucose level of the patient. The decisions on Meal and Exercises are almost instant and may change from one moment to another. They are always suitable for a particular person and in a particular situation: changes in glucose level can trigger new "reasoning" and provide new advice to the patient.

If our knowledge on reversibility of diabetes 2 will change in future, amendments to this application are minimal. The architecture from Figure 4 does not have to change because we allow an ad-hoc inference of object properties, as described in section 5.2. This type of flexibility is almost impossible to achieve with traditional and structured SQL databases: they are simply not made to carry out these types of data processing. We could have built a very big and rich SQL database to support decision making on diet and life style changes without reasoning, but the performance, efficiency and installation of such solutions would be almost impossible to achieve (27), particularly on smart devices. In short, all the flexibility of the proposed software solution will disappear if we move all our computations to traditional SQL databases.

We offer a high level of personalization of the reversibility of diabetes 2 . It allows constant changes in the way patient see the management of the chronic disease: patient could infer various constraints in the OWL ontologies in order to tailor the reversibility more towards their experiences of reacting to various recommended food and exercises. Without an ad-hoc inference, controlled by some OWL fixed constraints, the application would not be able to achieve this level of personalization. Particularly not, if we lose the opportunity to infer OWL concepts as much as possible.

\subsection{Future Work}

There are a few challenges, which should be resolved before the commercialization of this type of applications could start. Firstly, the weakest part of the architecture and its deployment are constant changes in open source software solutions and IDEs in particular, which will always affect the feasibility of the deployment of software architectures using technology specific components. However, as mentioned before, numerous plug-inns, which appear as we write, will probably be the answer for the future deployments of our solution. Secondly, OWLAPI is a backbone of our journey from the structured SQL processing to reasoning with SWRL and back: any problems associated with this particular API could affect the stability of applications generated from this architecture.

There is one aspect of our proposal, which has to be addressed in the very near future. It is applicable to the computations with SWRL enabled OWL ontologies. In order to prove the concept and achieve an overall goal of this research, we deliberately simplified the use of clinical data, i.e. glucose levels readings in particular, as a trigger for advising on changes in diets and life styles. It does not mean that this particular data is not sufficient for addressing the reversibility of diabetes 2 . On the contrary, many patients today are looking at instant values of their glucose levels, which can be taken or measured at their homes, in the morning, every day. These patients would use our application without any changes. However, there are more sophisticated ways of measuring response to glycemic index in anyone's diet and thus we should look at the way of addressing the reversibility of diabetes 2 through them. Our future OWL model should take into account all new research which exists in the field published in $(42,43,44)$. The ontology from Figure 6 could be expanded with new classes which will, together with adequate individuals and object properties, influence the reasoning process. In principle, they will filter individuals of Food into the Meal class, but according to a more sophisticated or more complicated criteria, then just simple taking into account the latest glucose level readings.

Finally, the ideas from this paper would fit and could be used within an initiative of addressing the role of Big Data in personalized medicine (45). The question of asserting essential individuals in OWL classes, asserting object properties, as in the applications presented here, and "feeding" any OWL ontology with relevant semantics, has been addressed in different publications $[46,47]$. This is a subject of a different debate in which we should 
discuss how to balance the level of inference and controlled assertions in the application. This is very important because we must secure the best possible reasoning for each patient who wishes to reverse diabetes 2 but would prefer to have a greater control on decision he/she can make in this process. Currently, we are able to feed OWL ontologies, without any restrictions with data from SQL like databases through assertions. If we wish to increase the level of inference we could do it within the Portege environment without any restrictions. In both cases the proposed architecture remains the same.

\section{References}

[1] R.J. Smith, "Personalized Medicine: Are we there yet?", Highlights from the Society of Medicines Research Symposium, October 2, 2014 - National Heart \& Lung Institute, Kensington, London, UK

[2] L. Mertz, "Ready or Not", IEEE Pulse, May/June 2014, pp. 45.47.

[3] D. Kalaitzopoulos "The Potential of Precision Medicine", in New Horizons in Translational Medicine, Volume 3, Issue 2, March 2016, pp 63-65.

[4] O.E. Streeter, P. J. Beron, P. N. Iyer "Precision Medicine : Genomic Profiles to Individualize Therapy" in Otolaryngology Clinics of North America, Available online on 17 May 2017, Elsevier.

[5] M.J. Khoury "The Shift from Personalized Medicine to Precision Medicine and Precision Public Health: Words Matter!" CDC Blogs, posted on April 2016, available at https://blogs.cdc.gov/genomics/2016/04/21/shift/

[6] "What is the Precision Medicine Initiative?", NIH Publication, Genetics Home Reference, Published on June 2017, available at https://ghr.nlm.nih.gov/primer/precisionmedicine/initiat ive

[7] G. De Micheli, C. Boero, C. Baj-Rossi, I. Taurino, S. Carrara "Integrated Biosensors for Personalized Medicine", in 49 ACM/EDAC/IEEE Conference on Design Automation Conference (DAC), 2012 49th ACM/EDAC/IEEE, 3-7 June 2012

[8]D. Johnson "Personalised Medicine Draws Closer with Cheap and Accurate DNA Sequences", in IEEE Spectrum, Posted, October 0216, available at http://spectrum.ieee.org/nanoclast/biomedical/devices/p ersonalized-medicine-draws-closer-with-cheap-andaccurate-dna-sequencer

[9] D.C Colins, R. Sundar, J.S.J. Lim, T.A. Yap “ Towards precision Medicine in the Clinic: From Biomarker Discovery to Novel Therapeutics", in Trends in Pharmacological Sciences, Special Issue on Precision Medicine, January 2017, Volume 38, No 1, pp 25-39

[10] L. Hood, M. Flores A personal view on systems medicine and the emergence of proactive $\mathrm{P} 4$ medicine: predictive, preventive, personalized and participatory, in New Biotechnology, Volume 29, Issue 6,15 September 2012, Pages 613-624
[11] A.P Dahawan, "Collaborative Paradigm of Preventive, Personalised and Precision Medicine with Point-ofCare Technologies" in IEEE Journal of Translational Engineering in Health and Medicine, December 2016

[12] A.P. Dhawan, W.J. Heetderks, M. Pavel, S. Acharya, M. Akay, AA. Mairal, B Wheeler, C. C. Dasco, T. Sunder, N. Lovell, M. Gerber, M. Shah, S.G. Senthilvei, M.D Wang, B. Bhargava "Current and Future Challenges in Point-of-Care Technologies: A Paradigm-Shift in Affordable Global Healthcare With Personalized and Preventive Medicine" in IEEE 2 IEEE Journal of Translational Engineering in Health and Medicine March 2015

[13]S. Bochia, "Why is Personalised Medicine relevant to Public Health, European Journal of Public Health, Vol 24, No 3, 2014, pp 249-350.

[14] H. Kondylakis, E.G Spanakis, S. Sfakianakis, V. Sakkalis, M. tsiknakis, K. marias, X. Zhao, H.Q.Yu, F. Dong, "Digital Patient: Personalized and translational data management through the MyHealthAvatar EU project" 37th Annual International Conference of the IEEE Engineering in Medicine and Biology Society (EMBS), Milano, Italy, August 25th-29th, 2015.

[15]E. Strickland, "Squishy Clockwork Biorobot Could Dose you with Drugs from the Inside", Blog posted on January 2017 at IEEE Spectrum, available at http://spectrum.ieee.org/the-humanos/biomedical/devices/squishy-clockwork-biobotcould-dose-you-with-drugs-from-the-inside

[16] D. Horgan, M. Ramao, R. Torbett, A. Brand, "European Data Driven Economy: A Lighthouse initiative on Personalised Medicine, in Health Policy and Technology, 2014, Volume 3, Published by Elsvier, pp. 226-233.

[17] R.W. Grant, D. J. Wexler, "Personalised Medicine in Type 2 diabetes: what does the future hold?" in Diabetes Mang (Lond). 2012, May; 2(3), pp 199-2014.

[18] R. Taylor, "Type 2 Diabetes, Diabetes Care, Volume 36, April 2013. Pp 1047-1055.

[19] P. Tusso, "Prediabetes and lifestye Modifictaion", Comentary in CME, The Premanente Journal, Summer 2014, Volume 18, Nuber 3, pp 88-92

[20] Zeevi, D., Korem, T., Zmora, Israeli, D., Rothschild, D., Weinberger, A., Ben-Yacov, O., Lador, D., AvnitSagi, T., Lotan-Pompan, M., Suez, J., Mahdi, J.A., Matot, E., Malka, G., Kosower, N., Rein, M., Zilberman-Schapira, G., Dohnalová, L., PevsnerFischer, M., Bikovsky, R.N., Halpern, Z., Elinav, E., Segal., E. (2015) Personalised Nutrition by Prediction of Glycemic responses, in Cell 163(5), November 2015, 1079-1094

[21] Y., Sun, W., You, F. Almeida, P., Estabrooks, B., Davy (2017) The effectovness and Cost of Lifestyle Interventions Including Nutrition Education for Diabettes Orevention: A Systematic Review and metaAnalysis in Journal of the Academy of Nutrition and Dietetics, March 2017, Volume 117, Number 3, pp. 404-421.

[22] R. Jumertz von Schwartzenberg, P.J. Turnbaugh, "Siri, What Should I Eat" Leading Edge Preview, in Cell 163, November 2015, pp 1051-1052. 
[23] W., Ahmad, N., Koay, R., Juric, R. (2011) The Management of the Relationships Between Symptoms, Diseases, Medicines and Treatments Using OWL and SWRL, in the Proceedings of the $16^{\text {th }}$ International Conference on System Design and Process Science, SDPS 2011, Jeju Island, Korea, June 2011

[24] N., Chammas, R., Juric, N., Koay, V., Gurupur, C.S., Suh, (2013) Towards a Software Tool for Raising Awareness of Diabetic Foot in Diabetic Patients, in the Proceedings of the international conference HICSS 46, Maui, Hawaii, January 2013

[25] P., Kataria, A., Macfie, R., Juric, K., Madani, (2008) Ontology for Supporting Context Aware Applications for the Intelligent Hospital Ward, in the Transaction of Integrated Design \& Process Science Tran-Disciplinary International Journal, August 2008, Vol. 12, No. 3, pp. 35-44.

[26] P., Kataria, R. Juric, (2010) "Sharing Healthcare Data through Ontological Layering" In Proceedings of the 43rd Annual Hawaii International Conference on System Sciences (HICSS 43), Kauai, Hawaii, January 5 $-8,2010$. IEEE, pp. 1-10.

[27] R., Saaidi, P., Kataria, R., Juric (2011) "Semantic Management of Submissions of Applications for Marketing Authorization of Medicines", book chapter, in: Suh, S.C., Tanik, M., Gurupur, V. P. (Eds.) Biomedical Engineering: Healthcare Systems, Technology and Techniques (Springer; 1st Edition (20 Aug 2011), pp.99-116.

[28] SWT Road Map, available at W3C website https://www.w3.org/2001/10/03-sww-1/slide7-0.html

[29] OWL 2 Web Ontology Language Profiles W3C Recommendation 2009 [Available from: https://www.w3.org/TR/2009/REC-owl2-profiles20091027

[30] SWRL: A Semantic Web Rule Language Combining OWL and RuleML 2004 [Available from: https://www.w3.org/Submission/SWRL/.

[31] B. Jafarpour, S.R Abidi, S. S. R. Abidi, "Exploiting Semantic Web Technologies to Develop OWL-Based Clinical Practice Guideline Execution Engines", in IEEE Journal of niomedicla and Health informatics, Vol 20, No 1, January 2016.

[32] X. Chen, N. Zhang, J. Chen, Z. Wu "OWL reasoning with Big Biomedical Data" in IEEE International Conference on Big Data, 2013

[33] M.M Romera, J.M. Vazquez-Naya, J. Pereira, M. Pereira, A. Pazos, G. Banos "The iOSC3 System: Using Ontologies and SWRL Rules for Intelligent Supervision and Care of Paitents with Acute Cardiac Disorder" in Computational and Mathematical Methods in Medicine, Volume 2013, January.

[34] C.T. Bau, R.C. Chen, C.Y Huang, "Construction of the clinical decision Support System for undergoing Surgery based on Domain Ontology and ruke Reasoning" in Telemedicine and e-health, May 2014

[35] S.Mekruksavanich."Medical Expert System Based Ontology for Diabetes Disease Diagnosis", in 7th IEEE International Conference on Software Engineering and Service Science (ICSESS), August, 2016.
[36] T. Pulkkinen, M. Sallimen, "Progressive monitoring and treatment planning of diabetes mellitus in smart home environment" in IEEE International Conference on Consumer Electronics (ICCE), January 2013. on

[37] I. Fiaz, H. Mukhtar, A-M. Qamar, S. Khan, "A Semantic Rules \& Reasoning based Approach for Diet and Exercise Management for Diabetets" in IEEE InternationL conference on Emerging Technologies, January 2014

[38] A. Arwan, B, Priyambadha, R. Sarno, M. Sidiq, H. Kristianto "Ontology and Semantic Matching for Diabetic Food Reccommendation", in IEEE International Conference on Information Technology and Electrical Engineering (ICITEE), December 2013.

[39] M.A. Basar, H.N. Alvi, G.N. Bokul, M.S. Khan, F. Anowar, M.N. Huda, K. A. Al Mamun "A review on Diabetes Paotent lifetye Managemnet Using Mobile Applications" in the Proceedings of the $18^{\text {th }}$ iNternational Conference on Computer and Information technology, December 2015

[40] R., Juric R \& I., Kim, I (2017) "Software Architectures for Smart Applications which Merge Ontological Reasoning with Big Data Analytics”. In the Proceedings of $21^{\text {st }}$ International Conference on System Design and Process Science, SDPS 2017, November 2017.

[41] K., Bravhar, R. Juric (2017)“Personalizing Drug Administration to Patients with Parkinson's Disease: Manipulating Sensor Generated Data in Android Environments", In Proceedings of the 50 $0^{\text {th }}$ HICSS 2017 Conference, January 2017.

[42]R.J. Smith, D.M. Nathan, S.A. Arslanian, L. Groop, R. A. Rizaa, J.I. Rotter " Individiualising Therapies in Type 2 Diabetes Mellitus Based on Paitent Characteristics: What we know and What we Need to Know, in JCEM, April 2010, Volume 95, Number 4 pp. 1566-1574

[43] D. Zeei, T. Korem, N. Zmora, Z. Halpern, E. Elinav, E. Segal "Personalised nutrition by Prediciton of Glycemic Response", in Cell 163, 1079-1094.

[44] P. Harrero, P. pesl, M. raddy, N. Oliver, P. Georgiou, C. Tpoumazou, "Advanced insulin bolus advisor based on run to run control and case based reasoning" in IEEE Journal of biomedical nh Health Informatics, Volume 9, Issue 3, May 2015, pp 1087-1096.

[45] M. Panahiazar, V. Taslimitehrani, A. Jadhav, J. Pathak, "Empowering Perosnalised Medicine with Big data and Semantic Web Technology: Promisses, Chalenges and Use Cases, in IEEE International Conference on Big Data, 2014.

[46] R., Juric (2017) Could Semantic Web Technologies Create New Computational Models outside Semantic Web, In the Proceedings of the $21^{\text {st }}$ International Conference on System Design and Process Science, SDPS 2017, December 2017.

[47] R., Shojanoori, R., Juric, M., Lohi (2012) Computationally Significant Semantics in Pervasive Healthcare, Journal of Integrated Design and Process Science, IOS Press, Volume 16, Number 1 - 2012, pp 43-62. 